\title{
A GENERALIZATION OF THE WEDDERBURN-ARTIN THEOREM
}

\author{
S. K. JAIN AND S. R. LÓPEZ-PERMOUTH \\ (Communicated by Donald S. Passman)
}

\begin{abstract}
The structure of rings such that each of its homomorphic images has the property that each cyclic right module over it is essentially embeddable in a direct summand is determined. Such rings are precisely (i) right uniserial rings, (ii) $n \times n$ matrix rings over two-sided uniserial rings with $n>1$, or (iii) sums of rings of the types (i) and (ii).
\end{abstract}

\section{INTRODUCTION}

In this paper we study rings $R$ with the following property (P): For all homomorphic images $\bar{R}$ of $R$, every cyclic right $\bar{R}$-module is essentially embeddable in a direct summand of $\bar{R}$. Our results generalize the celebrated WedderburnArtin theorem which characterizes rings $R$ such that over all the homomorphic images $\bar{R}$ the cyclic modules are isomorphic to direct summands of $\bar{R}$. Examples of rings satisfying $(\mathrm{P})$ include semisimple artinian rings and right uniserial rings. Indeed we show that a ring $R$ has property $(\mathrm{P})$ if and only if $R$ is a direct sum of right uniserial rings and matrix rings over right self-injective right uniserial rings if and only if $R$ is a semiperfect ring whose cyclic right modules are essentially embeddable in direct summands (Theorem 3.5). Throughout this paper, all rings have 1 and all modules are right unital, unless otherwise stated. By a right (left) uniserial ring, we mean a ring having a unique composition series of right (left) ideals. A ring which is both right and left uniserial will simply be called uniserial. A right uniserial ring is uniserial iff it is right self-injective. For any module $M, E(M), \operatorname{Soc}(M)$ and $J(M)$ will denote, respectively, the injective hull, the socle, and the Jacobson radical of $M$.

\section{Preliminary Results}

Throughout this section, we assume that $R$ is a ring satisfying property $(\mathrm{P})$.

\subsection{Lemma. $R$ is a semiperfect ring.}

Received by the editors March 7, 1988 and, in revised form, July 5, 1988.

1980 Mathematics Subject Classification (1985 Revision). Primary 16A48; Secondary 16A51, 16A52.

Key words and phrases. Essential submodules, right uniserial rings, self-injective rings. 
Proof. Let $N=$ prime radical of $R$ under our hypothesis, each right ideal of $R / N$ is an annihilator right ideal and hence $R$ is semiperfect [3, p. 204, Exercise 24.3(d)-(e)].

Since $R$ is semiperfect, $R$ has a complete orthogonal set $e_{1}, \ldots, e_{n}$ of idempotents such that, for all $i, e_{i} R e_{i}$ is a local ring. In the lemmas which follow the decomposition $R=e_{1} R \oplus \cdots \oplus e_{n} R$ will be frequently used. For $R$ modules $A$ and $B$, the notation $A \hookrightarrow^{\prime} B$ shall mean that $A$ is essentially embeddable in $B$.

2.2. Lemma. For $R=e_{1} R \oplus \cdots \oplus e_{n} R$, the following are true:

(i) $e_{i} R$ is uniform for all $i$,

(ii) Soc $R$ is essential in $R$, and

(iii) $R$ has Goldie dimension $n$.

Proof. Let $\mathbf{S}=\left\{S_{1}, \ldots, S_{k}\right\}$ be an irreduntant set of representatives for the simple $R$-modules and let $\mathbf{P}=\left\{e_{1} R, \ldots, e_{k} R\right\}$ be a complete set of representatives for the projective indecomposable $R$ modules.

Since every simple module $S$ is cyclic, it is essentially embeddable in $e R$ for some idempotent $e \in R$. Clearly $e R$ is indecomposable. Thus we can define a function $f: \mathbf{S} \rightarrow \mathbf{P}$ by $f\left(S_{i}\right)=e_{j} R$ where $S_{i} \hookrightarrow^{\prime} e_{j} R$. The function $f$ must be one to one, hence onto. It easily follows that each $e_{j} R(j=1, \ldots, n)$ contains an essential simple submodule $T_{j}$ and, therefore, each $e_{j} R$ is uniform. Also, $T_{1} \oplus \cdots \oplus T_{n}=\operatorname{Soc} R$ is essential in $R$. Thus $R$ has Goldie dimension $n$.

\subsection{Lemma. $R$ is right artinian.}

Proof. Clearly each cyclic $R$-module has nonzero socle. Thus, $R$ is left perfect because $R$ is semiperfect [2]. Furthermore, since $J(R) /(J(R))^{2}$ is completely reducible, $J(R) /(J(R))^{2}$ is embeddable in Soc $R$. This yields $J(R) /(J(R))^{2}$ is finitely generated and so $R$ is right artinian [1, p. 322].

2.4. Lemma. For $i \neq j$, let $e_{i} R$ and $e_{j} R$ be indecomposable summands of $R$. Then, either $e_{i} R$ is isomorphic to $e_{j} R$ or $\operatorname{Hom}_{R}\left(e_{i} R, e_{j} R\right)=0$.

Proof. Suppose $\sigma: e_{i} R \rightarrow e_{j} R$ is not zero, then $e_{i} R / \operatorname{Ker} \sigma$ is embeddable in $e_{j} R$. Since $e_{j} R$ is uniform (Lemma 2.2), such an embedding must be essential. This implies $E\left(e_{i} R / \operatorname{Ker} \sigma\right) \cong E\left(e_{j} R\right)$. Also, since $R$ satisfies property $(\mathrm{P})$ and it has Goldie dimension $n, E(R / \operatorname{Ker} \sigma) \cong E(R)$. Let $R=e_{1} R \oplus \cdots \oplus e_{n} R$. Then

$$
R / \operatorname{Ker} \sigma \cong e_{1} R \oplus \cdots \oplus e_{i} R / \operatorname{Ker} \sigma \oplus \cdots \oplus e_{j} R \oplus \cdots \oplus e_{n} R,
$$

which yields

$$
E\left(e_{1} R\right) \oplus \cdots \oplus E\left(e_{j} R\right) \oplus \cdots \oplus E\left(e_{j} R\right) \oplus \cdots \oplus E\left(e_{n} R\right)
$$

$\cong E(R / \operatorname{Ker} \sigma) \cong E(R) \cong E\left(e_{1} R\right) \oplus \cdots \oplus E\left(e_{i} R\right) \oplus \cdots \oplus E\left(e_{j} R\right) \oplus \cdots \oplus E\left(e_{n} R\right)$. 
Since $e_{k} R$ is uniform for all $k, E\left(e_{k} R\right)$ has local endomorphism ring. Hence from (1) $E\left(e_{i} R\right) \cong E\left(e_{j} R\right)$. But this implies that $E\left(e_{i} R\right)$ and $E\left(e_{j} R\right)$ contain isomorphic copies of the same simple submodule $S$ and, therefore, $e_{i} R$ and $e_{j} R$ both contain essentially a copy of $S$. This implies that $e_{i} R$ is isomorphic to $e_{j} R$.

2.5. Lemma. $R$ is a direct sum of matrix rings over local rings.

Proof. Let $\left[e_{i} R\right]=\sum e_{j} R$, where the $\sum$ runs over all $j$ for which $e_{j} R \cong e_{i} R$. Renumbering if necessary we may write

$$
R=\left[e_{1} R\right] \oplus \cdots \oplus\left[e_{k} R\right]
$$

where $k \leq n$. By Lemma 2.4, $\left[e_{1} R\right]$ is an ideal in $R$ and so

$$
R \cong M_{n_{1}}\left(e_{1} R e_{1}\right) \oplus \cdots \oplus M_{n_{k}}\left(e_{k} R e_{k}\right)
$$

where $n_{i}$ is the number of summands in $\left[e_{i} R\right]$.

Next we proceed to show that each local ring $e_{i} R e_{i}$ is indeed right uniserial.

2.6. Lemma. If $R=S_{n}$ is the $n \times n$ matrix ring over a local ring $S$, then $S$ is right uniserial.

Proof. Write $R=e_{11} R \oplus \cdots \oplus e_{n n} R$, where $e_{11}, e_{22}, \ldots, e_{n n}$ are the usual matrix units. Notice that each $e_{i i} R$ is indecomposable since $S$ is local.

Consider $I \subset e_{11} R$. Then $R / I \cong e_{11} R / I \times e_{22} R \times \cdots \times e_{n n} R$ is essentially embeddable in $R$ because the Goldie dimension of $R$ is $n$. Thus

$$
E(R / I) \cong E(R)
$$

and so

$$
E\left(e_{11} R / I\right) \times E\left(e_{22} R\right) \times \cdots \times E\left(e_{n n} R\right) \cong E\left(e_{11} R\right) \times E\left(e_{22} R\right) \times \cdots \times E\left(e_{n n} R\right) .
$$

Since $e_{i i} R$ is uniform (Lemma 2.2), $E\left(e_{i i} R\right)$ is also uniform. Therefore, by Azumaya diagram, $E\left(e_{11} R / I\right) \cong E\left(e_{11} R\right)$. This implies $e_{11} R / I$ is uniform. It follows that the submodules of $e_{11} R$ are linearly ordered. We show now that $S \cong e_{11} R e_{11}$ is right uniserial. Let $A, B$ be right ideals of $e_{11} R e_{11}$. Then $A e_{11} R \subset e_{11} R$ and $B e_{11} R \subset e_{11} R$ and so either $A e_{11} R \subset B e_{11} R$ or $B e_{11} R \subset$ $A e_{11} R$. But then either $A=A e_{11} R e_{11} \subset B e_{11} R e_{11}=B$ or $B=B e_{11} R e_{11} \subset$ $A e_{11} R e_{11}=A$, proving our assertion.

In the next section we shall obtain a characterization of rings with property $(\mathrm{P})$.

2.7. Remark. Note that in the proof of Lemmas $2.2-2.6$ we have only used that $R$ is a semiperfect ring each of whose cyclic $R$-modules is essentially embeddable in a direct summand of $R$. 


\section{Main Results}

We begin with

3.1. Theorem. Let $R$ be a ring with property $(\mathrm{P})$. Then $R$ is a direct sum of matrix rings over right uniserial rings.

Proof. The proof follows from Lemmas 2.5, 2.6, 2.7 and the fact that ring direct summands of a ring with property $(P)$ inherit the property $(P)$.

It is obvious that right uniserial rings have property $(P)$. In what follows we will concentrate on showing that for a right uniserial ring $S$, the matrix ring $R=S_{n}(n>1)$ satisfies property (P) if and only if $S$ is right self-injective. For the sake of our discussion we define property $(\mathrm{Q})$ for modules. We say that an $R$-module $M$ has property (Q) if each factor of $M$ is essentially embeddable in a direct summand of $M$.

3.2. Lemma. The $n \times n$ matrix ring over $R$ has property (Q) as a module over itself if and only if the $R$-module $R^{(n)}$ has property (Q).

Proof. Given a category isomorphism $F=\mathscr{M}_{S} \rightarrow \mathscr{M}_{T}$ between the categories of right modules of two rings $S$ and $T$, it is obvious that a module $M \in \mathscr{M}_{S}$ satisfies (Q) if and only if $F(M) \in \mathscr{M}_{T}$ satisfies (Q). Our lemma follows from the fact that if $e_{11} \in R_{n}$ is the usual matrix unit then $R^{(n)} \in \mathscr{M}_{R}$ corresponds to $R_{n} \in \mathscr{M}_{R_{n}}$ under the category isomorphism.

$$
-\otimes_{R_{n}} R_{n} e_{11}: \mathscr{M}_{R_{n}} \rightarrow \mathscr{M}_{R} \text {. }
$$

3.3. Lemma. If the $R$-module $R^{(n)}$ has property $(\mathrm{Q})$ where $R$ is right uniserial and $n>1$, then $R$ is right self-injective.

Proof. Let $R$ be a right uniserial ring which is not right self-injective. Then there exists $s \in R$ such that $x s \notin R x$. Without loss of generality, we may assume that $s$ is invertible. Define $I=(x,-x s, 0,0, \ldots, 0) R \subseteq R^{(n)}$. We claim that $R^{(n)} / I$ is not embeddable in $R^{(n)}$. Notice that both $\bar{e}_{1} R$ and $\bar{e}_{2} R$ are isomorphic to $R$ as $R$-modules, where $e_{1}=(1,0,0, \ldots, 0)$ and $e_{2}=$ $(0,1,0, \ldots, 0)$. Also, since $\bar{e}_{1} R \cap \bar{e}_{2} R=\bar{e}_{1} x R_{1}=\bar{e}_{2} x R$. If $\psi: R^{(n)} / I \rightarrow R^{(n)}$ were an embedding of $R^{(n)} / I$ into $R^{(n)}$, and if $\psi\left(\bar{e}_{1}\right)=\left(a_{1}, a_{2}, \ldots, a_{n}\right)$ and $\psi\left(\bar{e}_{2}\right)=\left(b_{1}, b_{2}, \ldots, b_{n}\right)$, then there must exist $i, j$ such that $a_{i}$ invertible and $b_{j}$ invertible. However, $\psi\left(\bar{e}_{1} x\right)=\left(a_{1} x, a_{2} x, \ldots, a_{n} x\right)$ and $\psi\left(\bar{e}_{2} x s\right)=$ $\left(b_{1} x s, b_{2} x s, \ldots, b_{n} x s\right)$, which implies that $a_{j} x=b_{j} x s$. Hence $b_{j}^{-1^{*}} a_{j} x=x s$, contradicting our choice of $s$. So we have shown that the $R$-module $R^{(n)}$ does not satisfy $(Q)$.

3.4. Lemma. If $R$ is a right self-injective right uniserial ring, then $R_{n}$ satisfies property $(\mathrm{P})$.

Proof. Since $R$ is self-injective, it follows that $R_{n}$ is also self-injective. Therefore. $R_{n}$ satisties property (Q) as a module over itself if and only if the injecrive hul! of an! cyclic $R_{n}$-module is embeddable in $R_{n}$. Let $e_{11} \in R_{n}$ be 
the usual matrix unit and let $I$ be a right ideal of $R_{n}$. Since $R_{n} \rightarrow R_{n} / I \rightarrow$ 0 is exact, $\left(R_{n} \otimes_{R_{n}} R_{n} e_{11}\right)_{R} \rightarrow\left(R_{n} / I \otimes_{R_{n}} R_{n} e_{11}\right)_{R} \rightarrow 0$ is also exact. But $\left(R_{n} \otimes_{R_{n}} R_{n} e_{11}\right)_{R} \cong\left(R_{n} e_{11}\right)_{R} \cong R^{(n)}$. Therefore, $N=R_{n} / I \otimes_{R_{n}} R_{n} e_{11}$ is a homomorphic image of $R^{(n)}$. Thus $N$ is an extension of a sum of $k$ cyclic $R$-modules, $(k \leq n)$ [5, Lemma 1.16]. But then, since $e_{11} R_{n}$ corresponds to $R$ under $\operatorname{Hom}_{R}\left(R_{n} e_{11},{ }_{-}\right)$, the inverse of $\left({ }_{-} \otimes_{R_{n}} R_{n} e_{11}\right)$, it follows that there exist $k$ quotients $Q_{1}, \ldots, Q_{k}$, of $e_{11} R_{n}$ such that $Q_{1} \oplus \cdots \oplus Q_{k} \hookrightarrow^{\prime} R_{n} / I$. Now, $E\left(Q_{i}\right) \hookrightarrow^{\prime} e_{11} R_{n}$ for all $i$. Hence $E\left(R_{n} / I\right)=E\left(Q_{1}\right) \oplus \cdots \oplus E\left(Q_{k}\right) \hookrightarrow^{\prime}$ $\left(e_{11} R_{n}\right)^{(k)} \hookrightarrow R_{n}$, proving that $E\left(R_{n} / I\right)$ is embeddable in $R_{n}$. Since each homomorphic image of $R$ is again right self-injective right uniserial, it follows that $R_{n}$ satisfies property $(\mathrm{P})$.

Our results are summarized in the following theorem.

3.5. Theorem. $A$ ring $R$ satisfies $(\mathrm{P})$ if and only if $R$ is a direct sum of right uniserial rings and matrix rings over right self-injective right uniserial rings if and only if $R$ is a semiperfect ring whose cyclics are essentially embeddable in a direct summand of $R$.

Proof. The proof follows from Theorem 3.1 and Lemmas 3.2, 3.3 and 3.4 and Remark 2.7.

\section{ACKNOWLEDGMENT}

The authors would like to thank Professor Carl Faith for pointing out that condition (1) in Exercise 24.3(e), page 204 in [3] should read "Every left ideal of $R / N$ is an annihilator left ideal."

\section{REFERENCES}

1. F. W. Anderson and Kent R. Fuller, Rings and categories of modules, Springer-Verlag, Berlin, Heidelberg and New York, 1974.

2. H. Bass, Finitistic dimension and a homological generalization of semiprimary rings, Trans. Amer. Math. Soc. 95 (1960), 466-488.

3. C. Faith, Algebra: Rings, modules and categories. II, Springer-Verlag, Berlin, Heidelberg and New York, 1976.

4. V. K. Goel and S. K. Jain, $\pi$-Injective modules and rings whose cyclics are $\pi$-injective, Comm. Algebra 6 (1978), 59-73.

5. B. L. Osofsky, Noncommutative rings whose cyclic modules have cyclic injective hulls, Pacific J. Math. 25 (1978), 331-340.

Department of Mathematics, Ohio University, Athens, Ohio 45701 